\title{
Métodos fisioterapêuticos utilizados na reabilitação do equilíbrio postural em indivíduos com osteoartrite: uma revisão sistemática
}

\author{
Physiotherapeutic methods used in the rehabilitation of the balance \\ postural in patients with osteoarthritis: a systematic review
}

Bruna Knob ${ }^{1}$, Matheus Santos Gomes Jorge ${ }^{1,2}$, Caroline Zanin ${ }^{1}$, Patrícia Rodigheri Vieira ${ }^{1}$, Willian Guerra de Lima ${ }^{1}$, Lia Mara Wibelinger ${ }^{1,2,3}$

\section{RESUMO}

A osteoartrite é uma doença que pode interferir no equilíbrio postural por meio de alterações neuromusculares diversas e a fisioterapia possui meios que buscam a manutenção ou reabilitação deste problema. O objetivo desta revisão foi identificar, na literatura, meios reabilitativos sobre o equilíbrio postural de indivíduos com osteoartrite. Realizou-se uma revisão sistemática nas bases de dados SciELO, Medline e LILACS, entre os meses de janeiro à março de 2016, a partir do descritor primário "Osteoartrite" (Osteoarthritis) em cruzamento com os descritores secundários "Equilíbrio Postural" (Postural Balance), "Fisioterapia" (Physical Therapy Specialty) e "Reabilitação" (Rehabilitation), nas línguas portuguesa e inglesa. Inicialmente encontraram-se 275 , dos quais 29 foram recrutados para uma análise qualitativa criteriosa. Ao fim, quatro artigos foram eleitos mediante os critérios estabelecidos pela revisão: um artigo abordou a cinesioterapia em solo, dois a hidrocinesioterapia e um a prática de exercícios domiciliares. Conclui-se que a manutenção ou reabilitação do equilíbrio postural, tanto estático quanto dinâmico, constitui um fenômeno de grande complexidade nos indivíduos acometidos pela osteoartrite. As técnicas fisioterapêuticas abordadas (cinesioterapia em solo, hidrocinesioterapia e a prática de exercícios físicos domiciliares) demonstraram efeitos positivos no manejo do equilíbrio postural em indivíduos com osteoartrite de quadril ou de joelho.

Palavras-chave: equilíbrio postural; osteoartrite; modalidades de fisioterapia; reabilitação.

\begin{abstract}
Osteoarthritis is a disease that can interfere with postural balance through various neuromuscular changes and physiotherapy has means that seek to maintain or rehabilitate this problem. The objective of this review was to identify, in the literature, rehabilitative means on the postural balance of individuals with osteoarthritis. A systematic review was performed between the months of January and March 2016 in the databases SciElo, Medline and LILACS, from the primary descriptor "Osteoartrite" (Osteoarthritis) in junction with the secondary keywords "Equilíbrio Postural" (Postural Balance), "Fisioterapia" (Physical Therapy Specialty) and "Reabilitação" (Rehabilitation) in Portuguese and English. Initially, 275 were found, of whom 29 were recruited for a qualitative analysis. Finally, four articles were chosen according to the criteria established by the review: one article addressed kinesiotherapy in soil, two to hydrokinesiotherapy and one to practice home exercises. It is concluded that the maintenance or rehabilitation of postural balance, both static and dynamic, is a phenomenon of great complexity in the individuals affected by osteoarthritis. The physiotherapeutic techniques (soil kinesiotherapy, hydrokinesiotherapy and physical exercises at home) showed positive effects on the management of postural balance in individuals with hip or knee osteoarthritis.
\end{abstract}

Keywords: postural balance; osteoarthritis; physical therapy modalities; rehabilitation.

Recebido em: 11/03/2017

Revisado em: 27/08/2017

Aprovado em: 13/11/2017

Autor para correspondência: Matheus Santos Gomes Jorge - Faculdade de Educação Física e Fisioterapia - Universidade de Passo Fundo - BR 285 São José - CEP: 99052-900 - Passo Fundo (RS), Brasil - E-mail: mathjorge5@gmail.com

Apoio financeiro: PRV recebeu bolsa do Pibic/UPF; WGL recebeu bolsa Pibic/CNPq, MSGJ recebeu bolsa Prosup/Capes

Conflito de interesses: nada a declarar. 


\section{INTRODUÇÃO}

A osteoartrite (OA) é uma doença articular inflamatória degenerativa resultante das respostas mediadas por condrócitos e sinoviócitos. Apresenta os níveis de soro e de citocinas inflamatórias sinoviais mais elevados do que em indivíduos não acometidos pela doença ${ }^{1,2}$. É a doença reumática mais prevalente na população geral, estando intimamente relacionada ao envelhecimento humano ${ }^{3}$.

Os achados clínicos incluem aumento do volume articular, dor ao repouso, rigidez matinal, fraqueza muscular, incapacidade, impacto na funcionalidade, entre outros agravos osteoarticulares ${ }^{4,5}$. Embora possa acometer quaisquer regiões do corpo, atinge preferencialmente as articulações que suportam grandes quantidades de peso e que requerem frequente utilização, como é o caso dos joelhos e do quadril ${ }^{2,6}$.

Os aspectos supracitados podem atuar como fatores desencadeantes para impacto em diversos âmbitos da saúde do indivíduo com OA, como a qualidade de vida e a propriocepção articular. Esta última afeta diretamente a estabilidade postural, fundamental para a mobilidade e atividades da vida diária, gerando alterações no equilíbrio postural (EP) e risco de quedas ${ }^{7-11}$.

Todavia, a fisioterapia parece ser uma estratégia no tratamento da OA, sobretudo a prática dos exercícios físicos de forma terapêutica. $\mathrm{O}$ exercício físico demonstra benefícios sobre o controle ou manejo da dor, redução da rigidez, aumento da mobilidade e da força muscular e melhora do EP destes indivíduos ${ }^{2,12,13}$.

Com base nas informações supracitadas, se faz necessária identificar na literatura os estudos que abordem o tema proposto, haja vista o pouco referencial de revisões sistemáticas acerca disso. Dessa forma, o objetivo do presente estudo foi revisar na literatura métodos fisioterapêuticos utilizados na reabilitação do EP em indivíduos com OA.

\section{MÉTODOS}

Trata-se de uma revisão sistemática desenvolvida a partir de uma consulta manual às bases de dados eletrônicos SciElo, Medline e LILACS, do período de janeiro à março de 2016. A busca teve como objetivo identificar artigos publicados entre os meses de janeiro de 2009 a março de 2016, a partir do descritor primário "Osteoartrite" (Osteoarthritis) que foi cruzado com os descritores secundários "Equilíbrio postural" (Postural Balance), "Fisioterapia" (Physical Therapy Specialty) e "Reabilitação" (Rehabilitation), nas línguas portuguesa e inglesa. Todos os descritores utilizados nesta revisão estavam indexador nos Descritores em Ciências da Saúde (DeCS).

Os bancos de dados utilizados para acessar as bases de dados foram o PubMed e a Bireme. Nestes foi possível aplicar a filtragem para seleção dos estudos, definindo-se previamente o tipo de estudo, o ano de publicação e os idiomas. Após aplicação dos filtros, os descritores foram cruzados da seguinte forma:

- "Osteoartrite" (Osteoarthritis) x "Equilíbrio postural" (Postural Balance) $\mathrm{x}$ "Fisioterapia" (Physical Therapy Specialty);

- "Osteoartrite" (Osteoarthritis) x "Equilíbrio postural" (Postural Balance) x "Reabilitação" (Rehabilitation).
- Os critérios de inclusão adotados na presente revisão foram:

- Estudos do tipo ensaios clínicos que poderiam ou não ser randomizados ou randomizados e controlados;

- Estudos descritos nas línguas portuguesa ou inglesa;

- Estudos que caracterizassem o tema "intervenção fisioterapêutica no EP em indivíduos com OA";

- Estudos que apresentaram em seu desfecho principal ou secundário o EP;

- Estudos realizados somente em humanos;

- Estudos disponíveis gratuitamente na íntegra nas bases de dados acima citadas.

- Mediante isso, foram definidos como critérios de exclusão:

- Estudos de outra natureza, tais como, revisões sistemáticas, estudos observacionais, estudos de caso ou estudos experimentais;

- Estudos abordassem em seu tema principal o tratamento medicamentoso ou cirúrgico;

- Estudos que não tenham sido concluídos;

- Estudos que apresentassem fuga do tema.

Com base no processo acima descrito, a Figura 1 apresenta a estratégia de busca e seleção dos artigos sobre os métodos fisioterapêuticos utilizados na reabilitação do $\mathrm{EP}$ em indivíduos com OA.

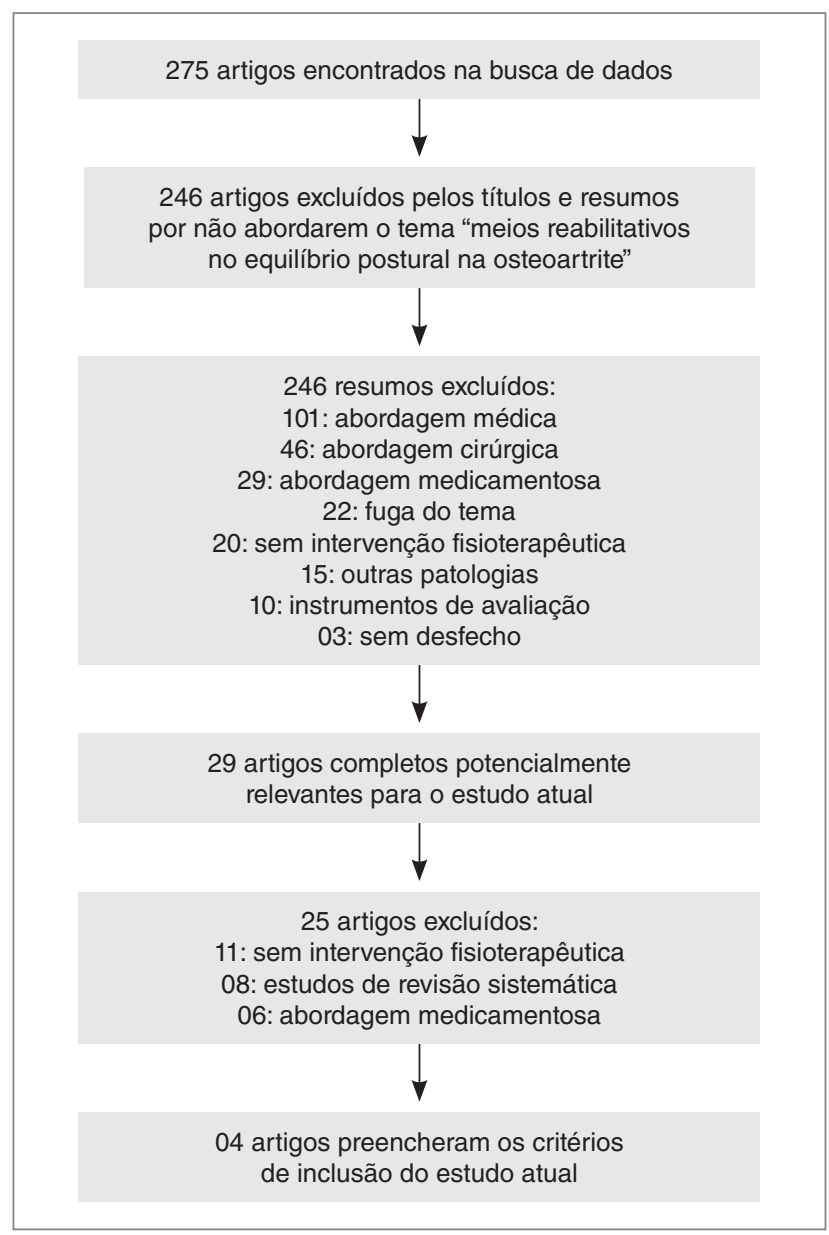

Figura 1: Fluxograma de estratégia de seleção dos artigos 
Encontraram-se 275 artigos, cujos mesmos tiveram seus títulos e resumos lidos por dois revisores independentes para assegurar a qualidade da busca. Em caso de dúvidas ou discordâncias um terceiro revisor era requisitado para analisar os artigos em questão.

De acordo com os critérios pré-estabelecidos, 29 artigos foram selecionados, os quais tiveram o seu conteúdo analisado. Para tanto, novamente, dois revisores independentes foram requisitados para tal tarefa e em caso de dúvidas ou discordâncias um terceiro revisor era requisitado para analisar os artigos em questão. Destes, quatro estudos que se enquadravam nos requisitos exigidos nessa revisão foram incluídos, permitindo a fundamentação teórica desta pesquisa.

\section{RESULTADOS}

Após o recrutamento dos estudos, todos foram avaliados qualitativamente pelos três revisores, por meio da leitura do seu conteúdo e extração dos dados necessários para compor a presente revisão. Estes dados foram organizados e montados conforme as tabelas seguintes.

A Tabela 1 apresenta os dados referenciais dos artigos selecionados. Observou-se que os estudos foram publicados nos últimos sete anos, sendo três em inglês e um em português. Os locais de origem dos periódicos onde os artigos foram publicados são América do Norte (02 artigos), América do Sul (01 artigo) e Europa (01 artigo). De acordo com a avaliação da Coordenação de Aperfeiçoamento de Pessoal de Ensino Superior do último quadriênio (CAPES), os artigos foram publicados em periódicos de boa qualidade ao analisar-se o qualis dos mesmos (A1, A2, B1 e B2) nas áreas adotadas pelos autores (Interdisciplinar e Educação Física). Os artigos foram localizados nas bases de dados Medline (03 artigos) e SciElo (01 artigo). Não foram localizados artigos na base de dados LILACS que se encaixassem dentro dos critérios exigidos. Tais informações são explanadas na Tabela 1.

Notou-se, que o tamanho amostral variou de 17 a 71 indivíduos com OA. Um dos estudos não especificou a média de idade dos participantes, todavia foi possível observar que as amostras dos demais estudos eram predominantemente compostas por idosos. Os locais de acometimento foram os joelhos e os quadris, sendo que um estudo envolveu ambas as articulações. Quanto ao sexo, dois estudos envolveram apenas mulheres, um estudo envolveu mulheres e homens e um estudo não especificou esta informação. Isso pode ser observado na Tabela 2.

Dentre os estudos selecionados, as intervenções fisioterapêuticas abordadas foram a cinesioterapia convencional (01 estudo), a hidrocinesioterapia (02 estudos) e os exercícios domiciliares (01 estudo). A maioria dos estudos compõe-se de uma combinação de modelos de intervenção e não uma técnica isolada. Isto é representado na Tabela 3 .

\section{DISCUSSÃO}

Com base nos temas abordados pelos estudos, percebe-se uma pequena variação entre as terapias selecionadas, como a cinesioterapia convencional, a hidrocinesioterapia e a prática de

Tabela 1: Caracterização dos dados referenciais dos artigos sobre os meios reabilitativos do equilíbrio postural na osteoartrite

\begin{tabular}{|c|c|c|c|c|c|}
\hline Autores, Ano & Título do artigo & Idioma & $\begin{array}{l}\text { Revista (país } \\
\text { de publicação) }\end{array}$ & $\begin{array}{c}\text { Qualis da } \\
\text { Revista }\end{array}$ & $\begin{array}{c}\text { Base } \\
\text { de dados }\end{array}$ \\
\hline $\begin{array}{l}\text { Rätsepsoo et al., } \\
2013^{11}\end{array}$ & $\begin{array}{l}\text { Leg extensor muscle strength, postural } \\
\text { stability, and fear of falling after a 2-month } \\
\text { home exercise program in women with } \\
\text { severe knee joint osteoarthritis }\end{array}$ & Inglês & $\begin{array}{l}\text { Medicina } \\
\text { (Kaunas) } \\
\text { (Holanda) }\end{array}$ & $\begin{array}{l}\text { B2 (área } \\
\text { Educação } \\
\text { Física) }\end{array}$ & Medline \\
\hline $\begin{array}{l}\text { Wibelinger et al,. } \\
2013^{12}\end{array}$ & $\begin{array}{l}\text { Efeitos da fisioterapia convencional e da witerapia } \\
\text { na dor e capacidade funcional de mulheres idosas } \\
\text { com osteoartrite de joelho }\end{array}$ & Português & $\begin{array}{l}\text { Revista Dor } \\
\text { (Brasil) }\end{array}$ & $\begin{array}{c}\text { B1 (área } \\
\text { Interdisciplinar) }\end{array}$ & SciELO \\
\hline $\begin{array}{l}\text { Arnold et al., } \\
2010^{13}\end{array}$ & $\begin{array}{l}\text { The relationship between falls efficacy and } \\
\text { improvement in fall risk factors following an } \\
\text { exercise plus educational intervention for older } \\
\text { adults with hip osteoarthritis }\end{array}$ & Inglês & $\begin{array}{l}\text { Physiotherapy } \\
\text { Canada } \\
\text { (Canadá) }\end{array}$ & $\begin{array}{c}\text { A2 (área } \\
\text { Interdisciplinar) }\end{array}$ & Medline \\
\hline $\begin{array}{l}\text { Hale, Waters e } \\
\text { Herbison, } 2012^{14}\end{array}$ & $\begin{array}{l}\text { A randomized controlled trial to investigate the } \\
\text { effects of water-based exercise to improve falls risk } \\
\text { and physical function in older adults with lower- } \\
\text { extremity osteoarthritis }\end{array}$ & Inglês & $\begin{array}{c}\text { Archives } \\
\text { of Physical } \\
\text { Medicine and } \\
\text { Rehabilitation } \\
\text { (Estados } \\
\text { Unidos) }\end{array}$ & $\begin{array}{c}\text { A1 (área } \\
\text { Interdisciplinar) }\end{array}$ & Medline \\
\hline
\end{tabular}

Tabela 2: Caracterização das amostras dos artigos sobre os meios reabilitativos do equilíbrio postural na osteoartrite

\begin{tabular}{|c|c|c|c|c|}
\hline Referência & Número & Idade & Local & Sexo \\
\hline Rätsepsoo et al., $2013^{11}$ & 17 indivíduos com osteoartrite & De 46 à 72 anos & Joelho unilateral & Mulheres \\
\hline Wibelinger et al., $2013^{12}$ & 71 indivíduos com osteoartrite & $\pm 66,7$ anos & Joelho & Mulheres \\
\hline Arnold et al., $2010^{13}$ & 54 indivíduos com osteoartrite & $\pm 73,7$ anos & Quadril & Não especificou \\
\hline Hale, Waters e Herbison, $2012^{14}$ & 39 indivíduos com osteoartrite & \pm 74 anos & Quadril ou joelho & $\begin{array}{c}26 \text { mulheres } \\
13 \text { homens }\end{array}$ \\
\hline
\end{tabular}


exercícios domiciliares. Assim, pelo fato do EP ser um componente que sofre influência de variados fatores físicos, ambientas e psicossociais, a consideração de todos eles para a escolha do melhor tratamento apresenta relevância.

A OA é uma condição articular crônica e a incidência de quedas entre seus portadores é alta, revelando a necessidade de medidas específicas para o seu tratamento ${ }^{7,14-16}$. As alterações neuromusculares, causadas pelo aumento da idade, contribuem para o comprometimento da dinâmica postural, reduzindo a capacidade do indivíduo de defender-se de possíveis quedas ${ }^{7}$. Além de justificar a escolha do tema proposto, as amostras dos estudos recrutados eram predominantemente idosas e observou-se que a fisioterapia foi benéfica para melhorar o EP em todos os estudos analisados ${ }^{12-15}$ e reduzir o risco de quedas em dois estudos ${ }^{14,15}$.

As quedas são uma condição de preocupação na área da saúde, com alto risco de ferimentos e impacto socioeconômico relevante. A possibilidade de evitar tal fenômeno depende de fatores posturais, ligados ao controle dinâmico do indivíduo $^{7}$, cujo mesmo pode ser reestabelecido pela fisioterapia em suas diferentes técnicas, citando-se a cinesioterapia ${ }^{13}$, a hidrocinesioterapia ${ }^{14,15}$ e a realização de exercícios domiciliares ${ }^{12}$, como foi observado na presente revisão sistemática.

Geralmente, o EP de indivíduos com OA assemelha-se ao de indivíduos sem a doença em atividades comuns, como sentar e/ou ficar em pé. Porém tende a sofrer alterações ${ }^{16}$ nas atividades de vida diária, como subir e/ou descer escadas, e nos escores físicos destes indivíduos, inclusive em estágios iniciais. Desta forma, ressalta-se a importância de intervenções precoces, como meio de manutenção do EP estático e dinâmico e da funcionalidade, evitando que tais déficits afetem futuramente a qualidade de vida ${ }^{17}$. Tais informações são confirmadas pelos estudos incluídos nesta revisão ${ }^{12-15}$, pois observou-se que, por meio dos diferentes métodos terapêuticos, foi possível reestabelecer o EP dos indivíduos com OA.

Indivíduos com OA apresentam maior oscilação no deslocamento no sentido anterior e posterior do corpo representando maior risco de quedas, especialmente aqueles que sofreram quedas anteriores. Dessa forma, as intervenções devem ser direcionadas especificamente à articulação instável que possa provocar alteração no $\mathrm{EP}^{18}$. O que concorda com a revisão atual, visto que os estudos selecionados direcionaram o

Tabela 3: Caracterização das avaliações e intervenções dos artigos sobre os meios reabilitativos do equilíbrio postural na osteoartrite

\begin{tabular}{|c|c|c|}
\hline Referência & Avaliações e intervenções & Desfechos/Resultados \\
\hline $\begin{array}{l}\text { Rätsepsoo et al., } \\
2013^{11}\end{array}$ & $\begin{array}{l}\text { - Avaliações: dinamômetro isocinético (força muscular), escala visual analógica (dor), } \\
\text { testes em plataformas de espuma (equilibrio e medo de quedas); } \\
\text { - Intervenção: exercícios domiciliares (fortalecimento com faixas elásticas, alongamentos } \\
\text { e exercícios de equilíbrio em steps) durante } 25 \text { min, associado ao acompanhamento } \\
\text { telefônico uma vez por semana. } \\
\text { - Tempo de intervenção: } 64 \text { sessões, } 7 x / \text { semana, } 02 \text { meses. }\end{array}$ & $\begin{array}{l}\text { Após a intervenção houve } \\
\text { aumento da força muscular e } \\
\text { do equilíbrio postural em bases } \\
\text { instáveis ( } p<0,05) \text {. As demais } \\
\text { variáveis não apresentaram } \\
\text { diferença significativa. }\end{array}$ \\
\hline $\begin{array}{l}\text { Wibelinger et al., } \\
2013^{12}\end{array}$ & $\begin{array}{l}\text { - Avaliações: Western Ontario and McMaster Osteoarthritis Index (rigidez, dor e } \\
\text { funcionalidade); Escala de Berg (equilíbrio). } \\
\text { - Intervenção G1 ( } n=33 \text { ): cinesioterapia convencional (exercícios respiratórios, } \\
\text { fortalecimento de membros inferiores com caneleiras } 500 \mathrm{~g} \text {, descarga de peso nos } \\
\text { membros inferiores, dissociação de cinturas em bola suiça, treino de marcha em solo, } \\
\text { rampas e escadas) durante } 50 \text { min. } \\
\text { - Intervenção } \mathrm{G} 2 \text { ( } \mathrm{n}=38 \text { ): cinesioterapia por meio do Nintendo Wii (exercícios de ioga, } \\
\text { exercícios respiratórios, exercícios de equilíbrio e propriocepção e atividades corporais) } \\
\text { durante } 30 \text { min. } \\
\text { - Tempo de intervenção: } 24 \text { sessões, } 2 x / \text { semana, } 03 \text { meses. }\end{array}$ & $\begin{array}{l}\text { Após a intervenção, o G1 } \\
\text { apresentou melhora da dor } \\
(p=0,00) \text { e da incapacidade } \\
(p=0,06) \text { e o } G 2 \text { apresentou } \\
\text { melhora da rigidez }(p=0,00) \text { e do } \\
\text { equilíbrio }(p=0,00)\end{array}$ \\
\hline $\begin{array}{l}\text { Arnold et al., } \\
2010^{13}\end{array}$ & $\begin{array}{l}\text { - Avaliações: Escala de Berg, Modified Clinical Test of Sensory Interaction and Balance } \\
\text { (equilíbrio), Activities-specific Balance Confidence Scale, Teste de levantar e caminhar } \\
\text { (risco de quedas), teste de caminhada de } 6 \text { minutos (velocidade e resistência). } \\
\text { - Intervenção G1 ( } \mathrm{n}=28) \text { : exercícios aquáticos (fortalecimento, controle do tronco, } \\
\text { equilíbrio e respiração) durante } 45 \text { min, associado à educação acerca da doença. } \\
\text { - Intervenção G2 ( } \mathrm{n}=26) \text { : exercícios aquáticos (fortalecimento, controle do tronco, } \\
\text { equilíbrio e respiração) durante } 45 \text { min. } \\
\text { - Tempo de intervenção do G1: } 22 \text { sessões, } 2 x / \text { semana, } 11 \text { semanas + programa } \\
\text { educacional } 1 \times / \text { semana. } \\
\text { - Tempo de intervenção do G2: } 22 \text { sessões, } 2 x / \text { semana, } 11 \text { semanas. }\end{array}$ & $\begin{array}{l}\text { Após a intervenção, o } \mathrm{G} 1 \\
\text { apresentou diminuição do } \\
\text { risco de quedas e aumento do } \\
\text { equilíbrio estatístico }(p<0,05) \text { e } \\
\text { o G2 não apresentou resultados } \\
\text { significativos. }\end{array}$ \\
\hline $\begin{array}{l}\text { Hale, Waters e } \\
\text { Herbison, } 2012^{14}\end{array}$ & $\begin{array}{l}\text { - Avaliações: Avaliação Física de Perfil Curto, Teste do Passo, Teste de levantar e } \\
\text { caminhar (risco de queda), Activities-specific Balance Confidence Scale (equilíbrio), } \\
\text { Western Ontario and McMaster Osteoarthritis Index (dor, rigidez, funcionalidade), Arthritis } \\
\text { Impact Measurement Scales 2-Short Form (impacto da doença). } \\
\text { - Intervenção G1 ( } \mathrm{n}=23 \text { ): exercícios aquáticos (aquecimento, alongamentos, } \\
\text { fortalecimentos, aeróbicos, exercícios de equilíbrio e propriocepção, treino de marcha e } \\
\text { relaxamento), durante } 20-60 \text { min. } \\
\text { - Intervenção G2 (n=16): exercícios domiciliares via web durante } 01 \text { hora. } \\
\text { - Tempo de intervenção do G1: } 24 \text { sessões, } 2 x / \text { semana, } 12 \text { semanas. } \\
\text { - Tempo de intervenção do G2: } 12 \text { sessões, } 1 \text { x/semana, } 12 \text { semanas. }\end{array}$ & $\begin{array}{l}\text { Após a intervenção, ambos os } \\
\text { grupos melhoraram os resultados } \\
\text { do Teste do Passo ( } \mathrm{G} 1 \mathrm{p}=0,000 \text { e } \\
\mathrm{G} 2 \mathrm{p}=0,002) \text { e o G2 apresentou } \\
\text { melhora da Avaliação Física de } \\
\text { Perfil Curto (tempo de reação } \\
\mathrm{p}=0,03 \text { e sensibilidade ao } \\
\text { contraste } \mathrm{p}=0,005=\text { menor risco } \\
\text { de quedas). Não houve melhora } \\
\text { diferença entre os grupos. }\end{array}$ \\
\hline
\end{tabular}

G1 (grupo 1); G2 (grupo 2) 
tratamento fisioterapêutico à duas articulações fundamentais no controle do EP: os joelhos e os quadris.

O EP dinâmico e a mobilidade adequada são fatores que podem influenciar no risco de quedas, porém não existem evidências de sua influência no EP estático ${ }^{16}$. Contudo, a prática fisioterapêutica busca melhor desempenho físico dos indivíduos acometidos pela OA, com a produção de reflexos na sua qualidade de vida ${ }^{17}$.

A perda de controle postural nos indivíduos com OA pode estar associada à fraqueza muscular, à redução dos mecanismos proprioceptivos e à limitação da funcionalidade, consequências estas que destacam a necessidade do envolvimento do tornozelo, do quadril e do treino de marcha no tratamento do EP de indivíduos com OA, especialmente através de exercícios cinesioterapêuticos ativos ${ }^{8,9,18}$. Dois estudos ${ }^{12,13}$ utilizaram técnicas cinesioterapêuticas ativas (exercícios de fortalecimento, propriocepção, equilíbrio, treino de marcha, alongamento), sendo que $\mathrm{um}^{12}$ realizou o tratamento domiciliar e outro ${ }^{13}$ utilizou a cinesioterapia por meio da wiiterapia (tratamento por meio de jogos eletrônicos). Ambos apresentaram resultados relevantes para a melhora do EP em indivíduos com OA.

A melhora da funcionalidade requer intervenções focadas no fortalecimento e no aumento na resistência muscular, especialmente de grupos musculares específicos, tais como o quadríceps femoral, os isquiossurais ou, ainda, os adutores e abdutores de quadril, cujos mesmos estão diretamente envolvidos no EP e no risco de quedas do indivíduo ${ }^{10,18}$. Nessa perspectiva, um estudo com 33 indivíduos com OA de joelho foram submetidos a exercícios de fortalecimento muscular, treino de propriocepção, equilíbrio e agilidade $(\mathrm{G} 1=8)$, treino de resistência física (G2=9), a combinação dos dois protocolos anteriores (G3) e massagem articular (G4 ou GC). Após 24 sessões, o G1, G2 e G3 apresentaram aumento da funcionalida$\mathrm{de}^{19,20}$, especialmente o G321. Reforçando as evidências de que os indivíduos com OA sejam beneficiados através da cinesioterapia ativa dos tecidos e que a melhora da funcionalidade do membro inferior esteja correlacionada com a melhora do EP nesta população.

O treinamento físico de exercícios de EP e de resistência física apresenta ganho funcional e redução do risco de quedas ${ }^{13}$. O que concorda com três dos estudos atuais ${ }^{12-14}$, visto que a prática física, tanto da cinesioterapia convencional, quanto a hidrocinesioterapia, envolveram exercícios de EP e resistência física e apresentaram melhora do EP e do risco de quedas. Contudo, um dos estudos selecionados ${ }^{15}$ que envolveu a prática hidrocinesioterapêutica e exercícios domiciliares não apresentou diferença significativa no desfecho do estudo.

Um dos estudos recrutados ${ }^{12}$ realizou um programa de 2 meses de exercícios domiciliares para fortalecimento dos músculos extensores do joelho. Ao final, observou-se o ganho de força muscular e de EP em bases instáveis, mas o EP em bases estáveis e o temor referido à queda não demonstrou diferença significativa entre o pré e o pós intervenção. Dessa forma, pode-se acreditar nos benefícios das orientações e práticas de atividades domiciliares como um complemento ao tratamento fisioterapêutico supervisionado e na sua parcela de sucesso do mesmo.

A prática hidrocinesioterapêutica e os exercícios aeróbicos mostraram-se benéficos no manejo do EP em indivíduos idosos com OA de joelho, mesmo longevos ${ }^{9,22}$. O que pode ser observado em dois de nossos estudos que abordaram a prática hidrocinesioterapêutica que envolveu exercícios resistidos e aeróbicos. Embora um dos estudos ${ }^{15}$ não tenha demonstrado significância estatística, o outro ${ }^{14}$ demonstrou que o exercício em água promoveu significativa melhora do equilíbrio de indivíduos com OA na fase pós intervenção.

A educação do indivíduo à autopromoção de saúde combinada ao plano de tratamento, oferta benefícios e contribui no manejo do $\mathrm{EP}$ em indivíduos com $\mathrm{OA}^{14}$. O que pode ser visto em dois estudos aqui recrutados ${ }^{12,14}$. Ambos envolveram a prática de exercícios domiciliares, sendo que um deles ${ }^{12}$ elaborou um plano de tratamento cinesioterapêutico para ser realizado em domicílio, já o outro ${ }^{14}$ incentivou a um plano de tratamento em domicílio em concomitância à exercícios aquáticos. Ambos os protocolos foram eficazes na melhora do EP em indivíduos com OA de joelho e de quadril.

Os dados encontrados acerca do assunto apresentam variações quanto ao benefício terapêutico dos exercícios, de acordo com o tempo e frequência da sua prática. É indispensável considerar os numerosos fatores envolvidos no processo patológico da OA, como o meio, aspectos psíquicos, sociais e físi$\cos ^{10,14}$. Os estudos não apresentam um consenso científico sobre a eficácia de algum método fisioterapêutico específico para o tratamento do equilíbrio em indivíduos com OA. Contudo, as evidências sugerem que o exercício terapêutico seja o meio mais promissor quanto a este assunto ${ }^{23}$. O que vem ao encontro da presente revisão, visto que três estudos recrutados ${ }^{12-14}$ envolveram a prática de exercícios físicos (em solo ou em meio aquático) e constataram efeitos significativos sobre o EP de indivíduos com OA.

Acredita-se que uma possível limitação desta revisão sistemática, seja o fato dos artigos não apresentarem a fase em que se encontra a doença (aguda ou crônica), nem os graus de OA envolvidos, salvo um dos estudos ${ }^{12}$ que demonstrou os efeitos da cinesioterapia convencional a domicilio em indivíduos com OA de joelho graus III e IV.

\section{CONCLUSÃO}

Em suma, as técnicas fisioterapêuticas abordadas pelos estudos, como a cinesioterapia em solo, a hidrocinesioterapia e a prática de exercícios físicos domiciliares demonstraram efeitos positivos no manejo do EP em indivíduos com OA. 


\section{REFERÊNCIAS}

1. Sohn DH, Sokolove J, Sharpe O, Erhart JC, Chandra PE, Lahey LJ, et al. Plasma proteins present in osteoarthritic synovial fluid can stimulate cytokine production via Toll-like receptor 4. Arthritis Res Ther. 2012;14(1):7.

http://dx.doi.org/10.1186/ar3555

2. Duarte VS, Santos ML, Rodrigues KA, Ramires JB, Arêas GPT, Borges GF. Exercícios físicos e osteoartrose: uma revisão sistemática. Fisioter Mov. 2013;26(1):193-202. http://dx.doi.org/10.1590/S0103-51502013000100022

3. Wibelinger LM. Fisioterapia em Reumatologia. $2^{\mathrm{a}}$ ed. Rio de Janeiro: Revinter, 2014.

4. Rezend MU, Campos GC. Is osteoarthritis a mechanical or inflammatory disease? Rev Bras Ortop. 2013;48(6):471-4. http://dx.doi.org/10.1016/j.rboe.2013.12.002

5. Jorge MSG, Zanin C, Knob B, Wibelinger. Efeitos do calor profundo no tratamento da dor na osteoartrite: revisão sistemática. Rev Dor. 2017; 18(1):79-84. http://dx.doi.org/10.5935/1806-0013.20170016

6. Choi YM, Dobson F, Martin J, Bennell KL, Hinman RS. Interrater and intrarater reliability of common clinical standing balance tests for people with hip osteoarthritis. Phys Ther. 2014;94(5):696-704. http://dx.doi.org/10.2522/ptj.20130266

7. Takacs J, Carpenter MG, Garland SJ, Hunt MA. The role of neuromuscular changes in aging and knee osteoarthritis on dynamic postural control. Aging Dis. 2013;4(2):84-99.

8. Khalaj N, Abu Osman NA, Mokhtar AH, George J, Abas WA. Effect of intra-articular hyaluronic injection on postural stability and risk of fall in patients with bilateral knee osteoarthritis. Scientific World J. 2014;2014: 815184

http://dx.doi.org/10.1155/2014/815184

9. Mat S, Tan MP, Kamaruzzaman SB, Ng CT. Physical therapies for improving balance and reducing falls risk in osteoarthritis of the knee: a systematic review. Age Ageing. 2015;44(1):16-24. http://dx.doi.org/10.1093/ageing/afu112

10. Silva A, Serrão PRMS, Driusso P, Mattiello SM. Efeito de exercícios terapêuticos no equilíbrio de mulheres com osteoartrite de joelho: uma revisão sistemática. Rev Bras Fisioter. 2012;16(1). http://dx.doi.org/10.1590/S1413-35552012000100002

11. Khalaj N, Abu Osman NA, Mokhtar AH, Mehdikhani M, Wan Abas WA. Balance and risk of fall in individuals with bilateral mild and moderate knee osteoarthritis. PLoS One. 2014;9(3): e92270. http://dx.doi.org/10.1371/journal.pone.0092270

12. Rätsepsoo M, Gapeyeva H, Sokk J, Ereline J, Haviko T, Pääsuke M. Leg extensor muscle strength, postural stability, and fear of falling after a 2-month home exercise program in women with severe knee joint osteoarthritis. Medicina (Kaunas). 2013;49(8):347-53.

13. Wibelinger LM, Batista JS, Vidmar MF, Kayser B, Pasqualotti A, Schneider $\mathrm{RH}$. Efeitos da fisioterapia convencional e da wiiterapia na dor e capacidade funcional de mulheres idosas com osteoartrite de joelho. Rev Dor. 2013;14(3):196-9.

http://dx.doi.org/10.1590/\$1806-00132013000300009

14. Arnold CM, Faulkner RA, Gyurcsik NC. The relationship between falls efficacy and improvement in fall risk factors following an exercise plus educational intervention for older adults with hip osteoarthritis. Physiother Can. 2011;63(4):41-420 http://dx.doi.org/10.3138/ptc.2010-29

15. Hale LA, Waters D, Herbison P. A randomized controlled trial to investigate the effects of water-based exercise to improve falls risk and physical function in older adults with lower-extremity osteoarthritis. Arch Phys Med Rehabil. 2012;93(1):27-34. http://dx.doi.org/10.1016/j.apmr.2011.08.004

16. Takacs J, Garland SJ, Carpenter MG, Hunt MA. Validity and reliability of the community balance and mobility scale in individuals with knee osteoarthritis. Phys Ther. 2014;94(6):866-74. http://dx.doi.org/10.2522/ptj.20130385

17. Reis JG, Gomes MM, Neves TM, Petrella M, Oliveira RDR, Abreu DCC Avaliação do controle postural e da qualidade de vida em idosas com osteoartrite de joelho. Rev Bras Reumatol. 2014;54(3):208-12. http://dx.doi.org/10.1016/j.rbr.2013.11.002

18. Petrella M, Neves TM, Reis JG, Gomes MM, Oliveira RDR, Abreu DCC. Parâmetros do controle postural em mulheres idosas com ou sem histórico de quedas associadas ou não à osteoartrite de joelhos. Rev Bras Reumatol. 2012;52(4):512-7. http://dx.doi.org/10.1590/S0482-50042012000400004

19. Sanchez-Ramirez DC, Van der Leeden M, Knol DL, Van der Esch M, Roorda LD, Verschueren S, et al. Association of postural control with muscle strength, proprioception, self-reported knee instability and activity limitations in patients with knee osteoarthritis. J Rehabil Med. 2013;45(2):192-7. http://dx.doi.org/10.2340/16501977-1087

20. Santos MLAS, Gomes WF, Queiroz BZ, Rosa NMB, Pereira DS, Dias JMD, et al. Desempenho muscular, dor, rigidez e funcionalidade de idosas com osteoartrite de joelho. Acta Ortop Bras. 2011;19(4):193-7. http://dx.doi.org/10.1590/S1413-78522011000400004

21. Rogers MW, Tamulevicius N, Semple SJ, Krkeljas Z. Efficacy of home-based kinesthesia, balance \& agility exercise training among persons with symptomatic knee osteoarthritis. J Sports Sci Med. 2012;11(4):751-8.

22. Wang $X Q$, Huang LY, Liu Y, Li JX, Wu X, Li HP, et al. Effects of tai chi program on neuromuscular function for patients with knee osteoarthritis: study protocol for a randomized controlled trial. Trials. 2013:14(1):37.

http://dx.doi.org/10.1186/1745-6215-14-375

23. Akyol Y, Durmus D, Alayli G, Tander B, Bek Y, Canturk F, et al Does short-wave diathermy increase the effectiveness of isokinetic exercise on pain, function, knee muscle strength, quality of life, and depression in the patients with knee osteoarthritis? A randomized controlled clinical study. Eur J Phys Rehabil Med. 2010;46(3):325-36. 